\title{
Statins exhibit anticancer effects through modifications of the pAkt signaling pathway
}

\author{
ERICA MIRAGLIA, JOHAN HÖGBERG and ULLA STENIUS \\ Institute of Environmental Medicine, Karolinska Institutet, S-171 77 Stockholm, Sweden
}

Received August 2, 2011; Accepted September 19, 2011

DOI: $10.3892 /$ ijo.2011.1223

\begin{abstract}
Statins are cholesterol lowering drugs that exhibit antitumor effects in several in vitro and in vivo models, and epidemiological studies indicate that statins prevent cancer. However, the molecular mechanism underlying the effects of statins still needs to be elucidated. We previously demonstrated that single doses of different statins rapidly affect Akt signaling via the purinergic receptor $\mathrm{P} 2 \mathrm{X} 7$. In particular, statins down-regulated nuclear pAkt. Here, we report that long-term treatment of A549 cells with high concentrations of statins (15-75 $\mu \mathrm{M})$ selects cell sub-populations exhibiting altered P2X receptor expression, signs of increased PTEN activity, enhanced PHLPP2, decreased PI3K p110 $\beta$ and inhibited downstream pAkt signaling. Furthermore, the nuclear accumulation of pAkt in response to insulin was inhibited in selected cells. Statin-selected cells displayed reduced proliferation rate and were more vulnerable to etoposide- and 5-fluorouracil-elicited cytotoxic effects. The stability of a selected phenotype $(50 \mu \mathrm{M})$ was tested for three weeks in the absence of statins. This resulted in a reversal of some, but not all alterations. Importantly, the truncated nuclear insulin response was retained. We conclude that long-term treatment with high doses of statins selects cells exhibiting stable alterations in insulin-Akt signaling and which are vulnerable to DNA damage. Our studies strengthen the hypothesis that an altered Akt signaling has a role in chemopreventive effects of statins.
\end{abstract}

\section{Introduction}

Statins are a class of drugs widely used in the treatment of hypercholesterolemia and in the prevention of cardiovascular diseases $(1,2)$. They are competitive inhibitors of 3-hydroxy-3-methylglutaryl-CoenzymeA (HMG-CoA) reductase, the rate-limiting enzyme in the mevalonate cascade, and attenuate the synthesis of cholesterol. Statins have a number of pleiotropic effects and not all of them can be related to an altered cholesterol metabolism

Correspondence to: Dr Ulla Stenius, Institute of Environmental Medicine, Karolinska Institutet, P.O. Box 210, 17177 Stockholm, Sweden

E-mail: ulla.stenius@ki.se

Key words: statins, pAkt, P2X7, cancer, chemoprevention
(3). In the last few years, the use of statins gained interest for cancer prevention $(4,5)$. Data using rodent models showed anticancer effects in myeloid leukemia and in colon, lung, prostate, breast, melanoma and glioma tumorigenesis (4-6). Use of statins was reported to be associated with significant reduction in risk of colon, lung, prostate, pancreas and esophagus cancer along with melanoma and B and T cell lymphoma (4,5,7-10). However, the exact mode of action of anti-proliferative and pro-apoptotic effects of statins still needs to be elucidated. The blockade of the mevalonate pathway may play an important role as it regulates the production of isoprenoid units, which are critical for the activation of Rho, Ras and Rab proteins (11). Additionally, statins regulate proliferation and apoptosis of tumor cells by affecting MAPK and Cdk2, which in turn reduce the expression of p21 and p27 cyclin kinase inhibitors (12). Further, statins were shown to induce derepression of PTEN expression via NFאB to inhibit breast cancer cell growth (6).

We previously demonstrated that statins rapidly activate the purinergic receptor $\mathrm{P} 2 \mathrm{X} 7$ and affect Akt signaling, which may have important anti-cancer effects (13-15): these effects were observed within few minutes, and were not prevented by pre-incubation with mevalonate. The Akt pathway is one of the major anti-apoptotic factors in cells (16): it is activated by growth factors and cellular stress and is commonly overexpressed in different tumors, but is also often induced by cytostatic treatment $(17,18)$. In addition, the PI3K/Akt signaling is necessary for many events of the metastatic pathway and has been implicated in the mechanisms of resistance to cytostatic drugs (19). Akt activation is antagonized by the lipid phosphatase PTEN (phosphatase and tensin homologue deleted on chromosome 10), which maintains the dynamic levels of the second messenger phosphatidylinositol 3,4,5-trisphosphate $\left(\mathrm{PIP}_{3}\right)$. Mutaton/deletion of PTEN has been reported in many cancers (20): therefore, the pAkt/PTEN axis is currently attracting great interest for its role in carcinogenesis. Moreover, we provided evidence for the involvement of PTEN, together with the protein phosphatases PHLPP1, PHLPP2, PP2A and calcineurin, in the rapid statin-induced $\mathrm{P} 2 \mathrm{X} 7$-mediated nuclear pAkt depletion (14).

The aim of this study was to investigate the long-term effects of high concentrations of statins in vitro and the possible role of the P2X7-Akt pathway in their anti-cancer properties. We focused on the long-term effects of statins because they mimic the conditions of chemopreventive strategies in vivo. Data are urgently needed to clarify the molecular mechanism(s) of the anti-cancer effects of statins in order to design safe and effective 
chemopreventive protocols; further, a better understanding of the mode of action of statins might provide essential information on what type of cancer can be prevented and the individuals who could benefit from the treatment. The prospect of reducing the incidence and burden of some of the most prevalent cancers with tolerable and affordable agents already used to prevent cardiovascular disease is encouraging but requires further exploration in clinical trials. Cancer preventive efforts with e.g., $\beta$-carotene and $\alpha$-tocopherol have failed in the past, and trials using retinoids for the prevention of advanced prostate cancer resulted in even negative effects (21). However, statins are the most promising agents available nowadays, and have been used for many years in successful cardiovascular disease prevention: thus a future use in cancer prevention cannot be expected to give unforeseen side effects.

In the present study we have investigated the effects of longterm atorvastatin-treatment on P2X7/Akt signaling. Our data show that long-term treatment selects cell populations with alterations in P2X7 and Akt pathway. Data indicate that statins can select cells with stable alterations in insulin-Akt signaling.

\section{Materials and methods}

Reagents. Atorvastatin, a synthetic statin characterized by high degree of lipophilicity, was kindly provided by Pfizer (New York, NY). Electrophoresis reagents were obtained from Bio-Rad Laboratories (Hercules, CA). The protein content of cell lysates was assessed with the Coomassie Brilliant Blue G-250 from Pierce (Rockford, IL). Etoposide, 5-fluorouracil, 3-(4,5-dimethylthiazol-2-yl)-2,5-diphenyltetrazolium bromide (MTT), adenosine 5'-triphosphate (ATP), 2'(3')-O-(4Benzoylbenzoyl)adenosine 5'-triphosphate (BzATP), KN62, Fura-2AM, insulin, mevalonate and all other reagents not specified here were purchased from Sigma Chemical Co. (St. Louis, MO). Atorvastatin, 5-fluorouracil, etoposide, BzATP and KN62 were dissolved in DMSO: the final concentration of DMSO added to the cells was $<0.2 \%$.

Cell culture. Non-small cell lung cancer (NSCLC) A549 cells were purchased from the American Type Culture Collection. A549 cells were grown in Dulbecco's modified Eagle's medium (DMEM), supplemented with $10 \%$ fetal calf serum (FCS), $1 \mathrm{mM}$ sodium pyruvate, $1 \%$ penicilline/streptomycin, and were maintained at $37^{\circ} \mathrm{C}$ in a humidified atmosphere of $5 \% \mathrm{CO}_{2}$. By culturing the parental cell line A549 in the presence of stepwise increasing concentrations of atorvastatin, we obtained five subpopulations which were resistant to different concentrations of statin. In this process, called the selection process, the medium was changed every $48 \mathrm{~h}$, and fresh statin was added. The concentration of atorvastatin in the medium was raised at every 4-5 passages. The selection process thus started with 4-5 passages at $15 \mu \mathrm{M}$ and then continued with $4-5$ passages at $30 \mu \mathrm{M}$ and so on. Cells were cultured under selection for at least 4 months. The selected sub-populations were named AS15, AS30, AS50, AS60, AS75 and were cultured in the presence of $15,30,50,60$ and $75 \mu \mathrm{M}$ atorvastatin, respectively, for further use. Serum-starved cells (for MTT assays and for experiments with insulin or cytostatic drugs) were obtained by culturing in DMEM supplemented with $0.1 \%$ FCS in the absence of atorvastatin for $24 \mathrm{~h}$.
Western blot analysis. Western blot analysis was performed as previously described (22). Briefly, the samples were subjected to SDS-PAGE and thereafter blotted onto a PVDF membrane (Bio-Rad). The protein bands were subsequently probed using antibodies against: P2X7, P2X4, NEDD4-1, $\beta$-catenin, phospho-Akt(Ser473), PTEN, PI3K p110 $\beta$, actin (from Santa Cruz, Santa Cruz, CA); PHLPP1 and PHLPP2 (from Bethyl Laboratoires); caveolin-1, NFkB p65, phospho-GSK33(Ser9), phospho-p70S6K(Thr389), phospho-FoxO1(Thr24)/3a(Thr32), phospho-mTOR(Ser2448), phosphor-IRS-1(Ser636/639) (Cell Signaling Technology, Beverly, MA). Proteins were detected by enhanced chemiluminescence (Amersham Biosciences, Uppsala, Sweden). The Western blot results were analyzed with NIH ImageJ 1.42 software.

Immunofluorescence staining. Cells were fixed in $3.7 \%$ formaldehyde for $20 \mathrm{~min}$. After fixation, cells were washed and stained with polyclonal antibodies against phospho-Akt(Thr308), $\beta$-catenin (Santa Cruz) and caveolin-1 (Cell Signaling Technology). After incubation with primary antibodies, secondary antibody conjugated with FITC (Dako, Glostrup, Denmark) was applied. No staining was detected when primary antibodies were omitted.

Cell viability and cell proliferation. The viability of cells was determined by the trypan blue exclusion assay. Cell proliferation was determined by MTT assay, which is based on the capacity of mitochondrial succinate dehydrogenase to convert MTT tetrazolium salt to formazan. Briefly, cells $\left(5 \times 10^{4}\right.$ cells per well) were seeded in 24-well plates. One day after seeding, cells were starved for $24 \mathrm{~h}$, and then treated with different compounds for $48 \mathrm{~h}$ (see Results). Afterwards, cells were incubated for $4 \mathrm{~h}$ with the medium containing MTT; then formazan was extracted from cells with DMSO, and absorbance was measured at $570 \mathrm{~nm}$.

Intracellular $\mathrm{Ca}^{2+}$ measurement. Cytosolic-free $\mathrm{Ca}^{2+}$ concentration was measured using the fluorescent indicator Fura-2AM. Briefly, cells were harvested and resuspended in Krebs-Ringer buffer $\left(125 \mathrm{mM} \mathrm{NaCl}, 5 \mathrm{mM} \mathrm{KCl}, 1.3 \mathrm{mM} \mathrm{CaCl}{ }_{2}, 1.2 \mathrm{mM}\right.$ $\mathrm{KH}_{2} \mathrm{PO}_{4}, 1.2 \mathrm{mM} \mathrm{MgSO}_{4}, 5 \mathrm{mM} \mathrm{NaHCO}, 25 \mathrm{mM}$ HEPES, $6 \mathrm{mM}$ glucose, and $2.5 \mathrm{mM}$ probenecid, $\mathrm{pH}$ 7.4) containing $5 \mu \mathrm{M}$ Fura-2AM. After a $30-\mathrm{min}$ incubation at $37^{\circ} \mathrm{C}$, the cell suspension was subjected to centrifugation and washed twice immediately prior to fluorescence measurement. Fluorescence was recorded using a Shimadzu RF-5000 spectrofluorometer; excitation wavelength was alternated between 340 and $380 \mathrm{~nm}$ and fluorescence intensity was monitored at an emission wavelength of $510 \mathrm{~nm}$ for at least $3 \mathrm{~min}$ after stimulation. The increase of intracellular $\mathrm{Ca}^{2+}$ upon agonist stimulation was calculated subtracting the ratio between the emitted signal at 340 and $380 \mathrm{~nm}$ in basal conditions from the ratio obtained after agonist stimulation, and is expressed as percentage of the basal ratio.

Statistical analysis. All the experiments were performed at least three times. Data are provided as the means \pm SEM. The results were analyzed by Student's t-test in the experiments comparing cell death and proliferation in the two cell sub-populations, and by one-way analysis of variance (ANOVA) and Bonferroni's test for all the other experiments (software: GraphPad Prism 5). $\mathrm{p}<0.05$ was considered significant. 
A

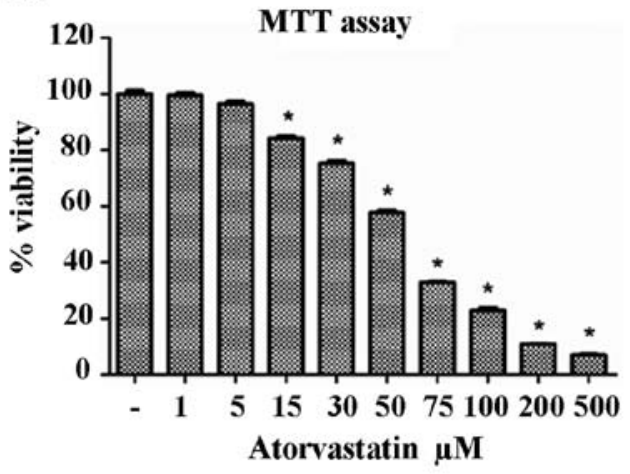

C

A549

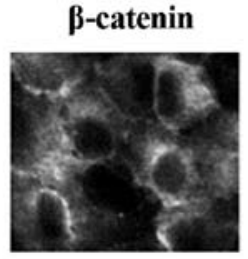

caveolin-1

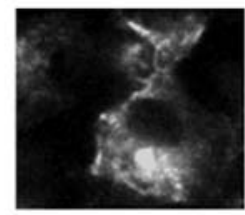

AS50
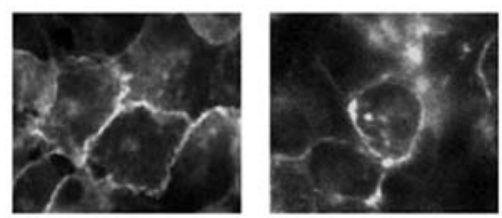

B
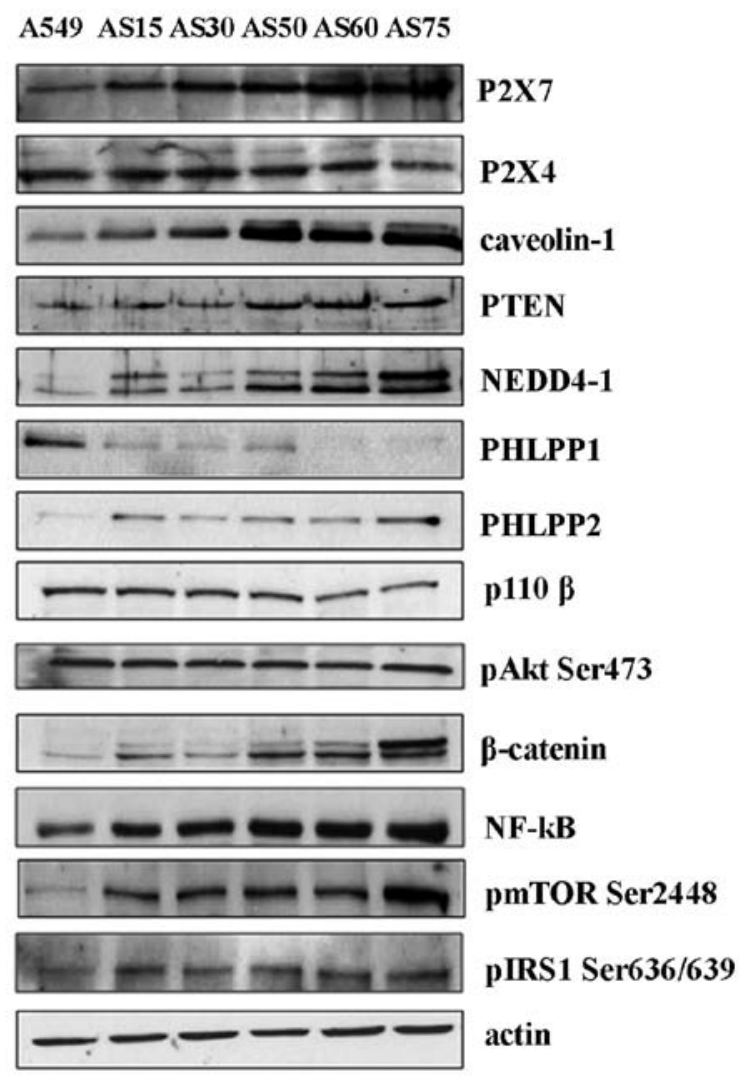

Figure 1. Atorvastatin long-term exposure selects cell sub-populations displaying altered signaling pathways. (A) A549 cells were exposed for $48 \mathrm{~h}$ to different concentrations of atorvastatin $(0-500 \mu \mathrm{M})$ to determine the cytotoxicity profile of this agent, assessed by MTT assay. The measurements were performed in triplicate, and data are presented as means \pm SEM $(n=3)$. Significance vs. A549: ${ }^{*}$ p $<0.001$. (B) A549 parental cells and the statin-selected cell sub-populations (AS15, AS30, AS50, AS60 and AS75), the latter cultured in the presence of the concentration of statin at which they were selected, were harvested 48 h after seeding and subjected to Western blot analysis. Actin levels were used as loading control. The figure is representative of 3 similar experiments. (C) The subcellular localization of $\beta$-catenin and caveolin- 1 was assessed by immunostaining in A549 parental cells and in the cell sub-population selected at $50 \mu \mathrm{M}$ atorvastatin (AS50). The figure is representative of 3 experiments with comparable results.

\section{Results}

Atorvastatin long-term treatment selects cell sub-populations expressing altered signaling pathways. Different types of statins have been reported to decrease the levels of phosphorylated Akt in several cell lines, and our group has shown that pravastatin, simvastatin and atorvastatin lower the insulininduced nuclear pAkt accumulation without affecting the total Akt levels in A549 cells (15). This effect is mediated by P2X7, and is associated with altered phosphorylations of downstream targets of pAkt, including GSK3 $\beta$ and cyclin D1 $(13,14)$. Here we investigated the cellular effects of the long-term treatment with atorvastatin to gain insight into their mode of action in the prevention of cancer. Atorvastatin-induced selection of A549 cells was studied as the short-term effects on Akt/P2X7 signaling are best characterized in this cell model $(13-15,22)$.

We first evaluated the cytotoxicity profile of atorvastatin on A549 cells. After $48 \mathrm{~h}$ of incubation with or without different concentrations of statin, ranging from 1 to $500 \mu \mathrm{M}$, cell viability was assessed by MTT assay. As reported in Fig. 1A, atorvastatin significantly affected cell viability already at $15 \mu \mathrm{M}(80 \%$ of the control) and the reduction of the number of cells was proportional to the concentration of this agent, with $<10 \%$ of viable cells in the presence of $500 \mu \mathrm{M}$ statin. The concentration of statin needed to halve the number of cells after $48 \mathrm{~h}$ ranged between 50 and $75 \mu \mathrm{M}$ (Fig. 1A).

In a first attempt of selection, atorvastatin was added to A549 cells at the concentration of $50 \mu \mathrm{M}$; with this approach most of the cells died and the surviving cells stopped to proliferate. Thus we decided to select the cells with stepwise increasing concentrations of statin, as described in Materials and methods. The first step in the selection process (4-5 passages at $15 \mu \mathrm{M})$ resulted in death for about $60 \%$ of the cells but surviving cells continued to grow. In the following steps this response pattern was repeated. The two highest concentrations of atorvastatin that permitted cell growth for several passages were 60 and $75 \mu \mathrm{M}$, but at these concentrations the growth rate was markedly reduced.

Five populations, i.e., AS15, AS30, AS50, AS60, AS75, were used for further studies. We have previously shown that the purinergic receptor $\mathrm{P} 2 \mathrm{X} 7$ mediates the rapid effects of statins on Akt activation. P2X7, along with $\mathrm{P} 2 \mathrm{X} 4$, is expressed in lung epithelial cells, and partly associates with lipid rafts $(23,24)$; these receptors have been demonstrated to form functional heterotrimers $(25,26)$. Moreover, caveolin-1 was shown to interact with $\mathrm{P} 2 \mathrm{X} 7$ and positively regulate its expression (23). Thus in the present study we investigated the expression of these proteins in atorvastatin-selected cells. We found a gradual increase of $\mathrm{P} 2 \mathrm{X} 7$, along with caveolin-1, during the 
A

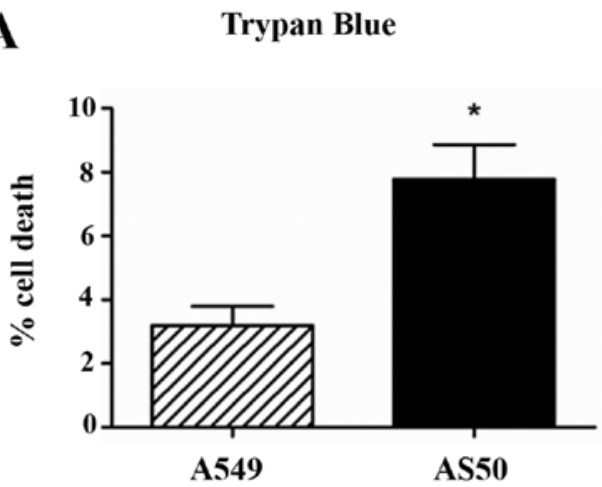

B

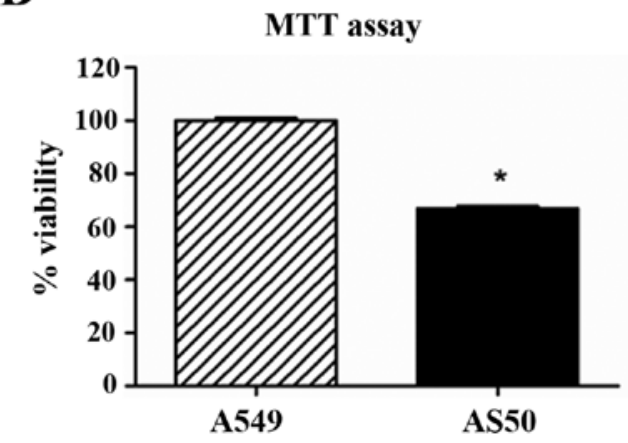

Figure 2. Statin-selected cells exhibit reduced cell viability and proliferation (A) A549 and atorvastatin-selected (AS50) cells were cultured in the absence or presence of atorvastatin for $72 \mathrm{~h}$, and the percentage of cell death was assessed with the trypan blue exclusion test. The measurements were performed in triplicate, and data are presented as means \pm SEM $(n=5)$. Significance vs. A549: " $\mathrm{p}<0.001$. (B) Starved A549 and AS50 cells were cultured in the absence or presence of atorvastatin for $48 \mathrm{~h}$, afterwards cell proliferation was assessed by MTT. The measurements were performed in triplicate, and data are presented as means \pm SEM $(n=4)$. Significance vs. A549: ${ }^{*} p<0.0001$.

selection process, and a parallel reduction of $\mathrm{P} 2 \mathrm{X} 4$ expression (Fig. 1B), indicating crosstalk and feedback signalling between P2X7 and P2X4 in atorvastatin-selected epithelial cells.

The lipid phosphatase PTEN is a negative regulator of Akt activation. PTEN expression was modulated in atorvastatin-selected cells, with a marked increase in the range of concentrations of 50-75 $\mu \mathrm{M}$ (Fig. 1B). Moreover, the expression of the protein NEDD4-1, the major E3 ubiquitin ligase regulating PTEN nuclear import (27), gradually augmented during the selection, with significantly higher levels already in AS15 cells (Fig. 1B).

The levels of the Ser/Thr protein phosphatases PHLPP1 and PHLPP2, which have been reported to dephosphorylate pAkt $(28,29)$, were also affected in the atorvastatin-treated subpopulations: PHLPP1 strongly decreased, almost disappeared, already in AS15 cells, whereas PHLPP2 expression markedly increased during the selection process, starting from the $15 \mu \mathrm{M}$ concentration (Fig. 1B). Interestingly, PHLPP1 and PHLPP2 selectively dephosphorylate specific Akt isoforms, thus differentially controlling the amplitude of Akt signaling: PHLPP1 has been suggested to play a role in glucose homeostasis, where Akt2 is critical, whereas PHLPP2 in cell survival, where Akt1 is critical (28).

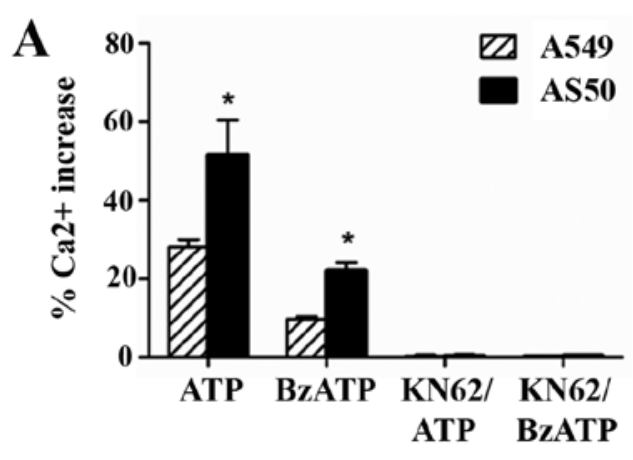

B

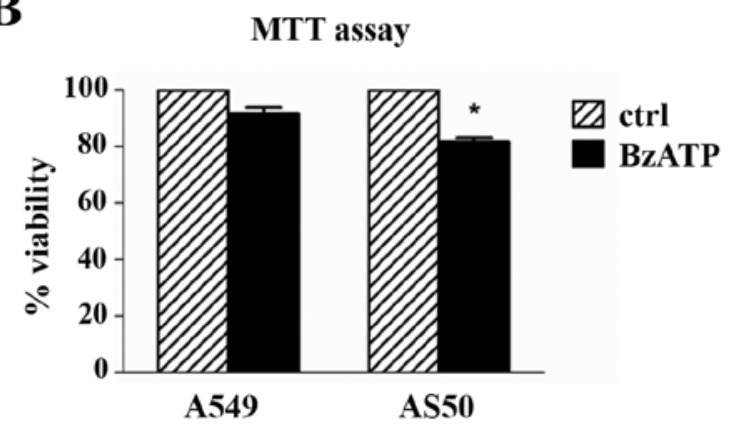

Figure 3. Statin-selected cells are more sensitive to agonists of the $\mathrm{P} 2 \mathrm{X} 7$ receptor. (A) A549 and atorvastatin-selected (AS50) cells were grown for $72 \mathrm{~h}$ in the absence or presence of atorvastatin; afterwards, the rise of cytosolic free $\mathrm{Ca}^{2+}$ upon agonist stimulation (ATP and BzATP, both at $100 \mu \mathrm{M}$ ), with or without pre-incubation with $\mathrm{KN} 62(100 \mathrm{nM}$, for $10 \mathrm{~min}$ ), was measured as described in Materials and methods. The figure is representative of 3 different experiments with comparable results. Significance vs. A549 in the same experimental condition: "p $<0.001$. (B) After starvation, cells were incubated for $48 \mathrm{~h}$ in the absence (ctrl) or presence of $100 \mu \mathrm{M}$ BzATP. Afterwards, MTT assay was carried out, and measurements were performed in triplicate. Results are expressed as percentage of the respective controls (untreated A549 or AS50 cells), which were assumed as $100 \%$, and are shown as means $\pm \operatorname{SEM}(\mathrm{n}=3)$. Significance vs. respective control: * $\mathrm{p}<0.001$.

Further, the levels of expression of the catalytic subunit of phosphoinositide 3-kinase $\mathrm{p} 110 \beta$, known to regulate nuclear Akt and affect cell proliferation (30), were assessed. As shown in Fig. 1B, the expression of this protein gradually decreased in statin long-term treated cells, with the lowest levels detected with the highest concentrations of statin tested. However, the levels of phosphorylated Akt were almost unaffected in statintreated cells in resting conditions (Fig. 1B).

Other important signaling pathways are likely to be modulated with chronic exposure to statins: the phosphorylation of the mammalian target of rapamycin (mTOR) progressively augmented in the cells under selection, along with phosphorylated insulin receptor substrate 1 (IRS-1), which increased at the $15 \mu \mathrm{M}$ concentration (Fig. 1B). The phosphorylation of FoxO1 and 3a, downstream targets of Akt, was diminished in the atorvastatin-selected sub-populations compared to the parental cells (Fig. 4A): this may cause nuclear translocation of these pro-apoptotic transcription factors, resulting in the growth arrest and activation of cell death programs. Further, the expression of the transcription factor $\mathrm{NFkB}$, which has been reported to differently modulate PTEN expression in 
A

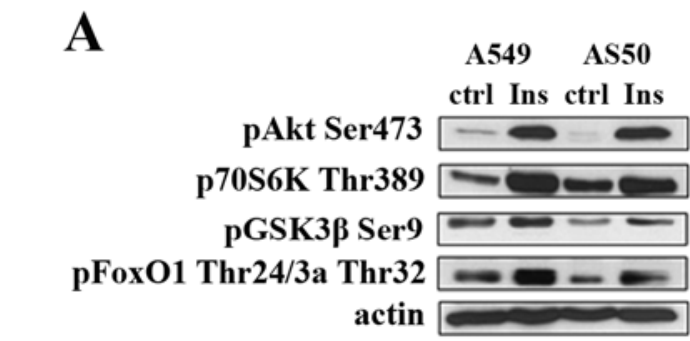

B

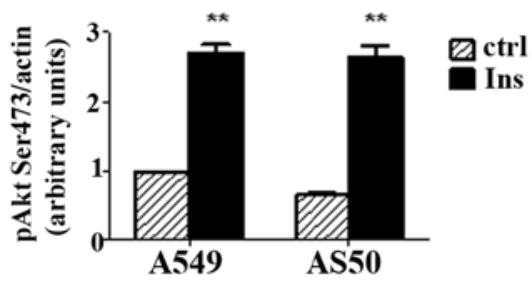

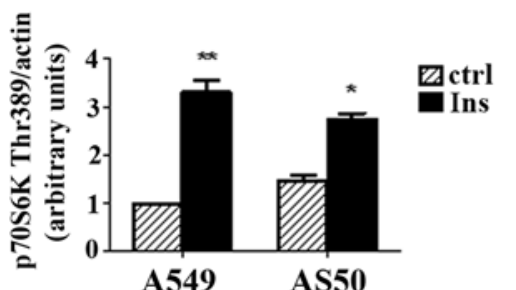

C

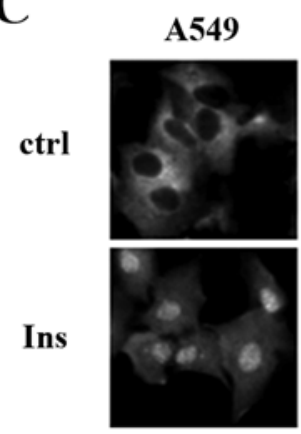

AS15

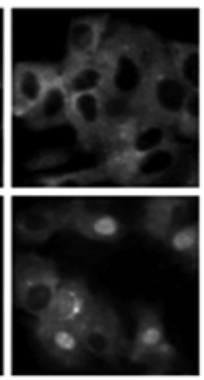

AS50

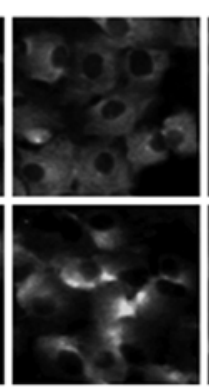

AS50+MEV

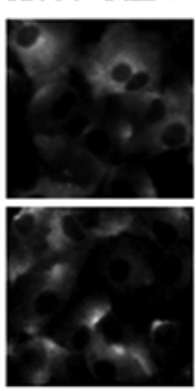

D

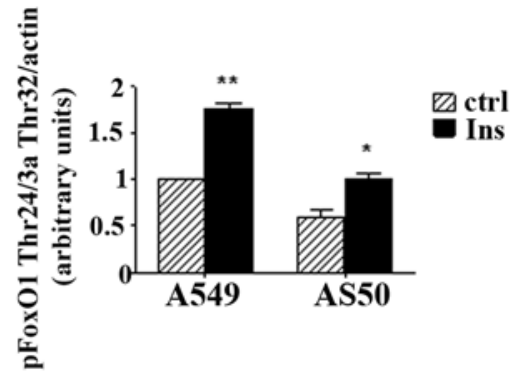

Figure 4. pAkt nuclear localization and its downstream signaling are inhibited in atorvastatin-selected cells. (A) A549 and atorvastatin-selected (AS50) cells were starved for $24 \mathrm{~h}$ in the absence of statin and then stimulated (Ins) or not (ctrl) with $1 \mu \mathrm{g} / \mathrm{ml}$ insulin for $15 \mathrm{~min}$. Cells were harvested and subjected to Western blot analysis. Actin levels were assessed as loading control. (B) Diagram shows the densitometric analysis of pAkt, pGSK3 $\beta$, p70S6K and pFoxol/3a from three independent experiments. Significance vs. corresponding control: ${ }^{*} \mathrm{p}<0.01 ;{ }^{* *} \mathrm{p}<0.001$. (C) A549, AS15 and AS50 cells, pre-treated or not for $24 \mathrm{~h}$ with $100 \mu \mathrm{M}$ mevalonate (AS50+MEV) were incubated for $15 \mathrm{~min}$ with (Ins) or without (ctrl) insulin. The insulin-response in terms of pAkt nuclear translocation was evaluated by immunofluorescence (C) and expressed as percentage of cells showing nuclear localization of pAkt (D). Significance vs. corresponding control: ${ }^{*} \mathrm{p}<0.001$.

different cell types $(6,31,32)$, was enhanced in the cells under selection with atorvastatin (Fig. 1B).

Finally, the long-term treatment with atorvastatin selected cells overexpressing $\beta$-catenin, as shown in Fig. 1B. $\beta$-catenin is a central component of the cadherin cell adhesion complex, maintaining the normal epithelial cell phenotype. A disturbance in epithelial cell adhesion, leading to a more invasive and metastatic phenotype, is a hallmark of tumor progression, and loss of $\beta$-catenin correlates with higher stage and grade in prostate carcinoma (33). Nevertheless, upon Wnt signaling pathway activation $\beta$-catenin can translocate to the nucleus and promote the transcription (34). To rule out the possibility of oncogenic activation of $\beta$-catenin in atorvastatin-selected cells we checked its cellular localization by immunofluorescence studies. Since the expression of the proteins involved in the P2X7-pAkt axis was most affected in the sub-populations obtained with the highest atorvastatin concentrations (50-75 $\mu \mathrm{M})$ we decided to employ AS50 cells in all the experiments of sub-cellular localization and to test the cellular response to different stimuli. $\beta$-catenin did not show nuclear translocation, but displayed a more limited and marked localization in the plasma membrane in AS50 cells (Fig. 1C). Incubation for $24 \mathrm{~h}$ with $100 \mu \mathrm{M}$ mevalonate did not modify this staining pattern, suggesting that these effects of statin were not merely a result of cholesterol or isoprenoid unit depletion (data not shown). Also caveolin-1 exhibited a more limited distribution to patches in the plasma membrane (Fig. 1C). This is in line with caveolin-1 localization in lipid rafts (35). No changes were observed for any of the other proteins whose expression was altered in atorvastatin-treated cells (data not shown).

Atorvastatin-selected cells exhibit lower viability and proliferation rate and are more sensitive to agonists of P2X7. AS50 cells were employed in further experiments to test their ability to respond to different stimuli. The percentage of dead cells, assessed by trypan blue exclusion test, was slightly increased in AS50 cells (Fig. 2A). In parallel, cell growth was measured by MTT assay, and it was remarkably reduced in the atorvastatinselected population compared to the parental cells (67\% of the control, Fig. 2B). No signs of autophagy (checked by Western blot analysis for the characteristic marker LC3 $\beta$ ) or apoptosis (assessed by Hoechst staining) were observed in atorvastatinselected cells (data not shown).

Since atorvastatin-selected cells displayed higher levels of $\mathrm{P} 2 \mathrm{X} 7$, we proceeded to characterize these cells for their responsiveness to ligands of the purinergic receptor. Both ATP 


\section{MTT assay}

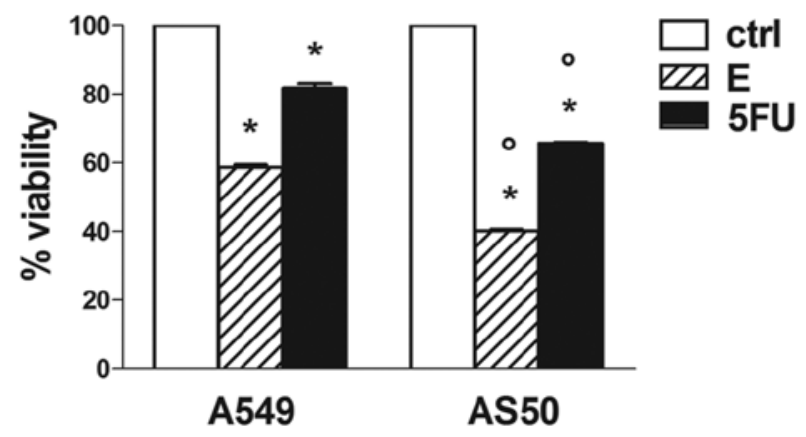

Figure 5. Atorvastatin-selected cells are more sensitive to cytostatic drugs. After starvation in the absence of statin, cells were incubated for $48 \mathrm{~h}$ in the absence (ctrl) or presence of $100 \mu \mathrm{M}$ etoposide (E) or $25 \mu \mathrm{M}$ 5-fluorouracil (5FU). Afterwards, cell viability was assessed by MTT assay. The measurements were performed in triplicate, and are shown as means \pm SEM $(n=3)$. Results are expressed as percentage of the respective controls (untreated A549 or AS50 cells). Significance vs. respective control: ${ }^{*} \mathrm{p}<0.0001$; significance vs. A549 in the same experimental condition: ${ }^{\circ} \mathrm{p}<0.0001$.

and BzATP triggered higher rise of the intracellular $\mathrm{Ca}^{2+}$ levels in AS50 than in A549 cells (Fig. 3A), and pre-incubation with the inhibitor of the $\mathrm{P} 2 \mathrm{X} 7$ receptor, KN62, completely blunted the agonist-elicited increase of free cytosolic $\mathrm{Ca}^{2+}$ in both the cells lines (Fig. 3A). Moreover, BzATP exerted a stronger effect on cell viability in atorvastatin-selected cells. Indeed, after exposure to BzATP for $48 \mathrm{~h}$ AS50 cells showed a reduced proliferation rate, measured by MTT assay, compared to parental cells, confirming the enhanced sensitivity of these cells to P2X7 stimulation (Fig. 3B).

Impairment of Akt signaling pathway in atorvastatin-selected cells. Atorvastatin-selected cells were then tested for their capability to respond to insulin, which is known to activate Akt in A549 cells (22). Cells were starved for $24 \mathrm{~h}$ to lower the basal pAkt levels and then stimulated with the hormone, and the cell lysates were subjected to Western blot analysis. The basal level of phosphorylation of Akt was slightly lower in AS50 cells compared to the parental cell line, but insulin elicited an increase of pAkt to the same level in both cell populations: this means that the increase of phosphorylated Akt was more pronounced in statin-selected cells than in the normal counterpart (Fig. 4A and B). The effect of insulin on some crucial downstream targets of Akt was also evaluated in statin-treated cells. p70S6K was higher in AS50 than in A549 cells in basal conditions, but upon insulin-stimulation we observed a reduced response in AS50 cells compared to the parental cells (Fig. 4A and B) GSK3 $\beta$ phosphorylation was significantly reduced in statin-selected cells, both in resting cells and after incubation with insulin. Further, Akt-mediated phosphorylation of the transcription factors FoxO1 and 3a was markedly decreased in the cells under selection, and the insulin-elicited response was remarkably reduced compared to the parental cells (Fig. 4A and B).

As an alternative approach, insulin-response was also assessed by immunofluorescence as induction of nuclear translocation of pAkt (22). The insulin-elicited accumulation of pAkt in the nucleus was slightly decreased in AS15 cells and completely absent in AS50 cells. Mevalonate failed to restore the response to insulin in AS50 cells, indicating that this effect of atorvastatin was mevalonate-independent in our model (Fig. 4C and D).

Statins have been shown to sensitize cancer cells to cytostatic drugs. For example, we have previously shown that short-term statin-treatment sensitize cells to cytostatic drugs $(13,15,22)$. Therefore we checked the responsiveness of long-term atorvastatin-selected cells to cytostatic drugs. Cells were incubated for $48 \mathrm{~h}$ in the absence or presence of $100 \mu \mathrm{M}$ etoposide or $25 \mu \mathrm{M}$ 5-fluorouracil, and viability was assessed by MTT assay. Effects on cell growth induced by both etoposide and 5-fluorouracil were more prominent in AS50 cells than A549 cells (Fig. 5), confirming the ability of statins to prime cells to cytostatic drugs.

The statin-induced alteration of protein expression is partially reverted after 3 weeks of statin withdrawal but insulin fails to trigger nuclear accumulation of pAkt. Lastly we evaluated the reversibility of the phenotype induced by selection with statin. AS50 cells were first cultured in medium deprived of atorvastatin for a period of time ranging from $24 \mathrm{~h}$ up to 10 days. Phosphorylated mTOR, which was augmented in AS50 cells, decreased to its basal level in $72 \mathrm{~h}$, as shown in Fig. 6A, whereas no changes were observed for all the other proteins analysed (data not shown). Therefore we checked the reversibility of the phenotype over a longer time period; when AS50 cells were cultured without atorvastatin for 3 weeks, PHLPP1 levels increased, showing an almost complete reversal (Fig. 6A). Also caveolin-1 and P2X 4 exhibited a similar timing, with caveolin-1 decreasing almost to the levels observed in parental cells and P2X4 slowly increasing after 3 weeks; instead, the expression of $\mathrm{P} 2 \mathrm{X} 7$ remained moderately high and did not return to the basal level observed in A549 cells (Fig. 6A). PTEN, which was induced in the selection process, was slightly diminished in AS50 cells grown in the absence of statin for 3 weeks, without reaching the lower levels of A549 cells (Fig. 6A). Also $\beta$-catenin expression, enhanced with the selection with statin, decreased to some extent, whereas PHLPP2 and NEDD4-1 levels remained high after 3 weeks of withdrawal (Fig. 6A). In the same way, p110 $\beta$ expression, which was decreased in statin-selected cells, did not return to the basal levels measured in the parental cells when AS50 cells were passaged for 3 weeks in the absence of statin. Finally, the expression of the transcription factor $\mathrm{NF} \mathrm{KB}$, which was induced in AS50 cells, decreased to the basal levels of A549 cells after 3 weeks of deprivation of statin (Fig. 6A).

The phenotype reversal was also checked in terms of capability of AS50 cells to respond to insulin after statin withdrawal. As shown in Fig. 6B and C, even after long-term (3 weeks) deprivation of atorvastatin insulin failed to induce pAkt nuclear localization in AS50 cells.

\section{Discussion}

Cell lines with acquired drug resistance frequently adapt cellular properties in response to the selection conditions. Thus, resistance models can provide information useful to understand the drug's mode of action. In this study we exposed A549 cells to increasing concentrations of atorvastatin, so that statin-resistant cells were selected in a stepwise fashion over a time period of 
A

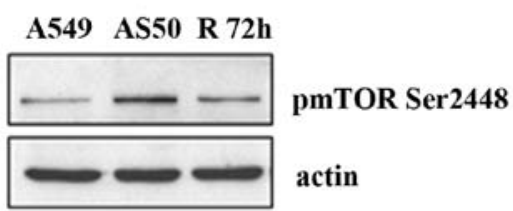

A549 AS50 R 3w

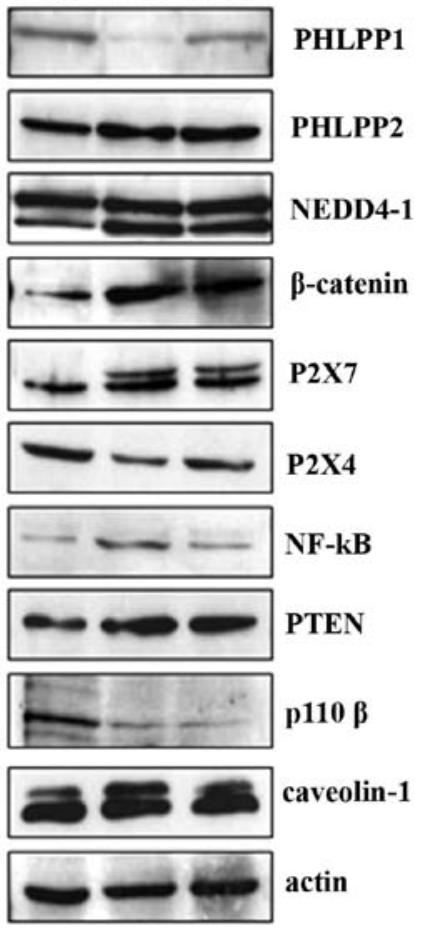

B

CTRL
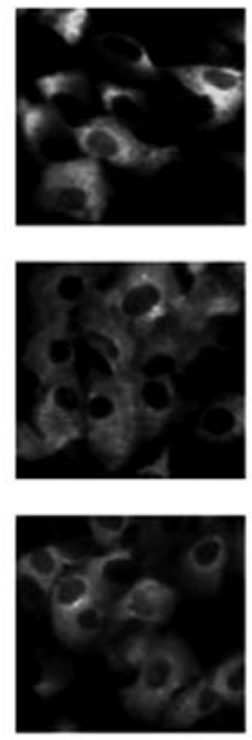

C

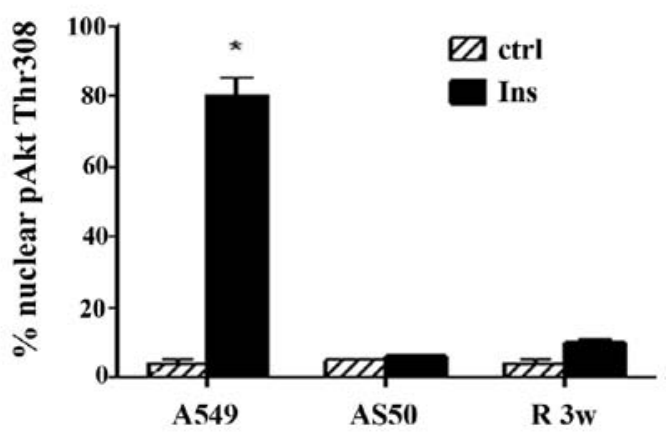

Figure 6. The statin selection-induced alterations of protein expression are partially reverted after 3 weeks of statin withdrawal but insulin fails to activate nuclear pAkt. (A) The reversal of the phenotype induced in statin-selected cells was assessed by Western blot analysis in atorvastatin-selected (AS50) cells that had been cultured in the absence of statin for $72 \mathrm{~h}(\mathrm{R} 72 \mathrm{~h}$ ) up to 3 weeks (R 3w). Actin levels were assessed as loading control. (B) Insulin-response was evaluated in AS50 cells after long-term statin withdrawal (3 weeks) by immunostaining for pAkt following insulin stimulation (B) and expressed as percentage of cells showing nuclear localization of pAkt (C). Significance vs. corresponding control: ${ }^{p} \mathrm{p}<0.001$.

several months. We find that the expression and at least some functions of $\mathrm{P} 2 \mathrm{X} 7$ are increased in these resistant cells. We also find that the downstream signaling of Akt is attenuated, and that the selection process resulted in an apparently stable cell phenotype exhibiting a reduced or absent nuclear pAkt response to insulin. These alterations confirm our previous results indicating that statins target the P2X7-Akt axis in cultured cells (13-15). Our present results also suggest that this axis is an important cell signaling pathway that might be critical for statin toxicity and that can modify insulin signaling.

Several of the observed alterations in protein expression can be seen as functional alterations that may result in a truncated insulin response, as manifested by the inhibition of pAkt accumulation in the nucleus. These include the up-regulated expression of PTEN, the reciprocal alterations in PHLPP1 and 2 levels, the up-regulation in mTOR activity, as well as the decreased p110 $\beta$ expression. These alterations may have participated in or caused the truncated insulin response. Some of these modifications were clearly seen in AS15 cells, therefore at a concentration not too far from the pharmacological concentrations employed in clinics, whereas other alterations were most marked in AS60 or AS75 cells, with high concentrations of drug. Other alterations can rather be seen as consequences of an inhibited accumulation of pAkt in the nucleus. These include the attenuated FoxO and GSK3 $\beta$ phosphorylations.

Can the monitored alterations confer resistance or be part of resistance development to statin toxicity? It seems reasonable to assume that an immediate downstream effect of P2X7 activation, such as the increased $\mathrm{P} 2 \mathrm{X} 7$-mediated $\mathrm{Ca}^{2+}$ release, was not responsible for statin toxicity. This response was increased in AS50 cells and the opposite should have been expected in case it was essential for resistance development. We thus assume that this response was not crucial for the selection. On the other hand, the insulin response, monitored as total increase of Akt phosphorylation, was not attenuated in AS50 cells, whereas the nuclear accumulation of pAkt was markedly decreased. This suggests that cytoplasmic pAkt was increased, and such an increase may have contributed to the resistance to statin toxicity. One possible target for cytoplasmic pAkt might have been I $\mathrm{B}$ kinase (IKK), with consequent induction of 
$\mathrm{NF} \mathrm{B}$, transcription factor promoting survival in response to various apoptotic stimuli (36).

It is not clear whether FoxO proteins are phosphorylated in the nucleus or in the cytoplasm, but our data showing inhibited FoxO phosphorylations lend some support for a phosphorylation mediated by nuclear pAkt. In any event, a decreased FoxO phosphorylation is regarded as pro-apoptotic event, but may also lead to protection against oxidative stress (37). Nuclear Akt may also affect cyclin D1 (14), and as we have discussed previously (14) such an effect may have prevented cell cycle progress and later apoptotic effects during cell cycle progression. The finding that DNA-damaging agents were more toxic in AS50 cells than in A549 cells supports an involvement of cell cycle alterations in the statin-induced resistance. The fact that the truncated insulin response became more pronounced in cells selected at higher concentrations of statin also argues in this direction. It may thus be suggested that the inhibited nuclear Akt response was crucial for the resistance against statins.

Further studies are needed to fully understand critical targets for the truncated insulin signaling. One possibility is that an mTOR-dependent feedback loop on pIRS-1 blunted PI3K activation (38). Another possibility is that the persistent up-regulation of $\mathrm{P} 2 \mathrm{X} 7$, perhaps in combination with alterations in other membrane proteins, in some way blocked the nuclear pAkt accumulation upon insulin stimulation. In other words it can be assumed, but remains to be shown, that the alterations in e.g., P2X7, P2X 4 and caveolin-1 induced by long-term treatment with high concentrations of statins may lead to aberrant signals that prevent insulin-elicited nuclear pAkt accumulation. Our earlier studies showed that statins, via P2X7, induce a coordinated but complex series of events that rapidly depleted nuclear pAkt levels (13-15). This suggests that statins may have a broader role in the regulation of nuclear pAkt than previously anticipated.

Regardless whether the truncated insulin response was critical for the selection or not, it seems to be a rare type of alteration. To our knowledge a similar response to xenobiotics or other pathogenic agents has not been previously described. The finding that the selected phenotype was not reverted by mevalonate suggests that it was not a consequence of cholesterol depletion. Alternatively, it may have been induced by cholesterol depletion, but eventually resulted in epigenetic alterations that made the reversal impossible. A third possibility is that we selected for a mutation already present in a minority of the A549 cells, and that it conferred resistance against toxicity by preventing nuclear pAkt accumulation. In any event our present and previous data suggest that regulation of nuclear pAkt expression is complex and that both low and high or toxic concentrations of statins can down-regulate it, albeit via different mechanisms and within vastly different time frames.

The intracellular localization of Akt is crucial for its activity: Akt undergoes nuclear translocation upon mitogenic activation (39), and constitutively activated nuclear Akt was found in hepatocellular HepG2 cells but not in normal liver cells, suggesting that its presence might be an important contributor in cell transformation (40). Phosphorylated Akt has been shown to be overexpressed in endometrial carcinoma compared to benign proliferative and secretory endometrium (41), and may have a critical role in e.g., prostate cancer development (42); further, the expression of p1 $10 \beta$ correlates with prostate cancer aggres- siveness (43). Evidence for a chemopreventive effect of statins in this tumor type is also the most convincing evidence that has been published (5). Our data thus add to previous data and corroborate a role for $\mathrm{P} 2 \mathrm{X} 7$ and nuclear pAkt in the not yet characterized mechanism for the ability of statins to prevent prostate cancer. Our data may also have a bearing on recent clinical data suggesting that statins may induce type 2 diabetes (44), as they indicate that long-term treatment may lead to a truncated insulin response. Further, the rare adverse effect leading to rhabdomyolysis may also relate to a diminished nuclear Akt activity. This side effect is more common in women and it has been shown that nuclear Akt in muscle cells is regulated by estrogen (45), so we might speculate that women may be more sensitive to decreased levels of nuclear Akt.

In conclusion, long-term exposure of statins selects cell subpopulations with altered insulin-Akt signaling. Furthermore, statin-selected cells were found to have reduced proliferation rate and increased sensitivity to cytostatic drugs. This study supports a role for altered Akt signaling in chemopreventive effects of statins.

\section{Acknowledgements}

This work was supported by Karolinska Institutet Funds.

\section{References}

1. Shepherd J, Cobbe SM, Ford I, et al: Prevention of coronary heart disease with pravastatin in men with hypercholesterolemia. West of Scotland Coronary Prevention Study Group. N Engl J Med 333: 1301-1307, 1995.

2. Collins R, Armitage J, Parish S, et al: Heart Protection Study Collaborative Group. MRC/BHF Heart Protection Study of cholesterol-lowering with simvastatin in 5963 people with diabetes: a randomised placebo-controlled trial. Lancet 361: 2005-2016, 2003.

3. Solomon KR and Freeman MR: Do the cholesterol-lowering properties of statins affect cancer risk? Trends Endocrinol Metab 19: 113-121, 2008.

4. Demierre MF, Higgins PD, Gruber SB, et al: Statins and cancer prevention. Nat Rev Cancer 5: 930-942, 2005.

5. Platz EA, Leitzmann MF, Visvanathan K, et al: Statin drugs and risk of advanced prostate cancer. J Natl Cancer Inst 98: 1819-1825, 2006.

6. Ghosh-Choudhury N, Mandal CC, Ghosh-Choudhury N, et al: Simvastatin induces derepression of PTEN expression via NFkappaB to inhibit breast cancer cell growth. Cell Signal 22: 749-758, 2010

7. Dellavalle RP, Nicholas MK and Schilling LM: Melanoma chemoprevention: a role for statins or fibrates? Am J Ther 10: 203-210, 2003.

8. Poynter JN, Gruber SB, Higgins PD, et al: Statins and the risk of colorectal cancer. N Engl J Med 352: 2184-2192, 2005.

9. Hamilton RJ and Freedland SJ: Review of recent evidence in support of a role for statins in the prevention of prostate cancer. Curr Opin Urol 18: 333-339, 2008.

10. Khurana V, Sheth A, Caldito G and Barkin JS: Statins reduce the risk of pancreatic cancer in humans: a case-control study of half a million veterans. Pancreas 34: 260-265, 2007.

11. Brunsveld L, Kuhlmann J, Alexandrov K, et al: Lipidated ras and rab peptides and proteins - synthesis, structure, and function. Angew C http://www.hlektronikotsigaro.gr/ hem Int Ed Engl 45: 6622-6646, 2006.

12. Denoyelle C, Vasse M, Körner M, et al: Cerivastatin, an inhibitor of HMG-CoA reductase, inhibits the signaling pathways involved in the invasiveness and metastatic properties of highly invasive breast cancer cell lines: an in vitro study. Carcinogenesis 22: 1139-1148, 2001.

13. Mistafa O, Hogberg J and Stenius U: Statins and ATP regulate nuclear pAkt via the P2X7 purinergic receptor in epithelial cells. Biochem Biophys Res Commun 365: 131-136, 2008. 
14. Mistafa O, Ghalali A, Kadekar S, Högberg J and Stenius U: Purinergic receptor-mediated rapid depletion of nuclear phosphorylated Akt depends on pleckstrin homology domain leucine-rich repeat phosphatase, calcineurin, protein phosphatase $2 \mathrm{~A}$, and PTEN phosphatases. J Biol Chem 285: 27900-27910, 2010.

15. Mistafa $\mathrm{O}$ and Stenius U: Statins inhibit Akt/PKB signaling via $\mathrm{P} 2 \mathrm{X} 7$ receptor in pancreatic cancer cells. Biochem Pharmacol 78: 1115-1126, 2009

16. Fresno Vara JA, Casado E, De Castro J, et al: PI3K/Akt signalling pathway and cancer. Cancer Treat Rev 30: 193-204, 2004.

17. Vivanco I and Sawyers CL: The phosphatidylinositol 3-Kinase AKT pathway in human cancer. Nat Rev Cancer 2: 489-501, 2002.

18. West KA, Castillo SS and Dennis PA: Activation of the PI3K/Akt pathway and chemotherapeutic resistance. Drug Resist Updat 5: 234-248, 2002

19. Qiao M, Sheng S and Pardee AB: Metastasis and AKT activation. Cell Cycle 7: 2991-2996, 2008.

20. Salmena L, Carracedo A and Pandolfi PP: Tenets of PTEN tumor suppression. Cell 133: 403-414, 2008.

21. Freemantle SJ, Spinella MJ and Dmitrovsky E: Retinoids in cancer therapy and chemoprevention: promise meets resistance. Oncogene 22: 7305-7315, 2003.

22. Roudier E, Mistafa O and Stenius U: Statins induce mammalian target of rapamycin (mTOR)-mediated inhibition of Akt signalling and sensitize p53-deficient cells to cytostatic drugs. Mol Cancer Ther 5: 2706-2715, 2006.

23. Barth K, Weinhold K, Guenther A, et al: Caveolin-1 influences P2X7 receptor expression and localization in mouse lung alveolar epithelial cells. FEBS J 274: 3021-3033, 2007.

24. Barth K, Weinhold K, Guenther A, et al: Characterization of the molecular interaction between caveolin- 1 and the $\mathrm{P} 2 \mathrm{X}$ receptors 4 and 7 in E10 mouse lung alveolar epithelial cells. Int J Biochem Cell Biol 40: 2230-2239, 2008.

25. Guo C, Masin M, Qureshi OS and Murrell-Lagnado RD: Evidence for functional P2X 4/P2X7 heteromeric receptors. Mol Pharmacol 72: 1447-1456, 2007

26. Casas-Pruneda G, Reyes JP, Pérez-Flores G, et al: Functional interactions between $\mathrm{P} 2 \mathrm{X} 4$ and $\mathrm{P} 2 \mathrm{X} 7$ receptors from mouse salivary epithelia. J Physiol 587: 2887-2901, 2009.

27. Wang X, Trotman LC, Koppie T, et al: NEDD4-1 is a protooncogenic ubiquitin ligase for PTEN. Cell 128: 129-139, 2007.

28. Brognard J, Sierecki E, Gao T and Newton AC: PHLPP and second isoform, PHLPP2, differentially attenuate the amplitude of Akt signaling by regulating distinct Akt isoforms. Mol Cell 25: 917-931, 2007

29. Gao T, Furnari F and Newton AC: PHLPP: a phosphatase that directly dephosphorylates Akt, promotes apoptosis, and suppresses tumor growth. Mol Cell 18: 13-24, 2005.

30. Marqués M, Kumar A, Poveda AM, et al: Specific function of phosphoinositide 3-kinase beta in the control of DNA replication. Proc Natl Acad Sci USA 106: 7525-7530, 2009.
31. Chandrasekar B, Boylston WH, Venkatachalam K, et al: Adiponectin blocks interleukin-18-mediated endothelial cell death via APPL1-dependent AMP-activated protein kinase (AMPK) activation and IKK/NF-kappaB/PTEN suppression. J Biol Chem 283: 24889-24898, 2008.

32. Zabalgoitia M, Colston JT, Reddy SV, et al: Carbon monoxide donors or heme oxygenase-1 (HO-1) overexpression blocks interleukin-18-mediated NF-kappaB-PTEN-dependent human cardiac endothelial cell death. Free Radic Biol Med 44: 284-298, 2008.

33. Pontes J Jr, Srougi M, Borra PM, et al: E-cadherin and beta-catenin loss of expression related to bone metastasis in prostate cancer. Appl Immunohistochem Mol Morphol 18: 179-184, 2010.

34. Cadigan KM: Wnt-beta-catenin signalling. Curr Biol 18: R943R947, 2008.

35. Michel V and Bakovic M: Lipid rafts in health and disease. Biol Cell 99: 129-140, 2007.

36. Romashkova JA and Makarov SS: NF-kappaB is a target of AKT in anti-apoptotic PDGF signalling. Nature 401: 86-90, 1999.

37. Greer EL and Brunet A: FOXO transcription factors at the interface between longevity and tumor suppression. Oncogene 24 : 7410-7425, 2005

38. Veilleux A, Houde VP, Bellmann K and Marette A: Chronic inhibition of the mTORC1/S6K1 pathway increases insulin-induced PI3K activity but inhibits Akt2 and glucose transport stimulation in 3T3-L1 adipocytes. Mol Endocrinol 24: 766-778, 2010

39. Meier R, Alessi DR, Cron P, et al: Mitogenic activation, phosphorylation, and nuclear translocation of protein kinase $\mathrm{B} \beta$. J Biol Chem 272: 30491-30497, 1997.

40. Syed NA, Horner KN, Misra V and Khandelwal RL: Different cellular localization, translocation, and insulin-induced phosphorylation of $\mathrm{PKB} \alpha$ in HepG2 cells and hepatocytes. J Cell Biochem 86: 118-127, 2002.

41. Shen Q, Stanton ML, Feng W, et al: Morphoproteomic analysis reveals an overexpressed and constitutively activated phospholipase D1-mTORC2 pathway in endometrial carcinoma. Int J Clin Exp Pathol 4: 13-21, 2010.

42. Trotman LC, Alimonti A, Scaglioni PP, et al: Identification of a tumour suppressor network opposing nuclear Akt function. Nature 441: 523-527, 2006.

43. Zhu Q, Youn H, Tang J, et al: Phosphoinositide 3-OH kinase p85alpha and p110beta are essential for androgen receptor transactivation and tumor progression in prostate cancers. Oncogene 27: 4569-4579, 2008

44. Rajpathak SN, Kumbhani DJ, Crandall J, et al: Statin therapy and risk of developing type 2 diabetes: a meta-analysis. Diabetes Care 32: 1924-1929, 2009.

45. Vasconsuelo A, Milanesi L and Boland R: 17Beta-estradiol abrogates apoptosis in murine skeletal muscle cells through estrogen receptors: role of the phosphatidylinositol 3-kinase/Akt pathway. J Endocrinol 196: 385-397, 2008. 